\title{
Zur Unmöglichkeit des Managements von Spitälern - die Paradoxie von Management
}

\author{
Beginn einer Reihe mit Beiträgen zum Spitalmanagement. Der erste Teil zeigt die \\ Paradoxie eines Managements auf, das trotz vielfältiger Entscheidungsformen auto- \\ nomer Teilbereiche für spitalweite Entscheidungen sorgen muss. Die Möglichkeiten \\ eines solchen Managements sind Thema des zweiten und dritten Beitrags in den \\ folgenden Ausgaben der SÄZ.
}

Harald Tuckermann ${ }^{a}$, Johannes Rüegg-Stürm ${ }^{b}$, Matthias Mitterlechnerc

a Prof. Dr. oec. HSG, Assistenzprofessor für Management pluralistischer Organisationen und Co-Leiter Forschungsprogramm HealthCare Excellence, Vize-Direktor des Instituts für Systemisches Management und Public Governance an der Universität St. Gallen

b Prof. Dr. oec. HSG, Direktor des Instituts für Systemisches Management und Public

Governance an de

Universität St. Gallen

c Dr. oec. HSG, Habilitand und Co-Leiter Forschungsprogramm HealthCare Excellence an der Universität St. Gallen

Korrespondenz:

Prof. Dr. oec.

Johannes Rüegg-Stürm Institut für Systemisches Management und Public Governance Dufourstrasse 40 a CH-9008 St. Gallen Tel. 0712242323 Fax 0712242536

johannes.rueegg[at]unisg.ch
Nimmt man Spitäler als Organisationen näher in den Blick, kann man den Eindruck gewinnen, dass Management als Funktion dieser Organisationen eine schier unmögliche Aufgabe darstellt: Auf der einen Seite beherbergen Spitäler viele medizinische Disziplinen, der Pflege und den sogenannten administrativen Bereichen drei Professionen mit einer Vielzahl unterschiedlicher Interessen, Perspektiven und Verständnissen. Auf der anderen Seite steigt mit dieser Vielfalt der Bedarf an organisationsweiten Entscheidungen. Zwischen Disziplinen und Fachbereichen stellen sich verstärkt Fragen der Koordination und Abstimmung innerhalb von Patientenprozessen, sowie mit Blick auf die für die Patientenbehandlung notwendigen Ressourcen, seien es Personal, Information, technische Ausstattung oder Räumlichkeiten. Mit der Einführung der DRGs, der viel diskutierten Spitalliste oder dem Wegfall der Kantonsgrenzen in der Gesundheitsversorgung kommen weitere organisationsweite Fragestellungen hinzu.

Zwischen organisationsweiten Fragestellungen und der Vielfalt von Professionen und Disziplinen entsteht ein Spannungsfeld, in dem Entscheidungen unmöglich oder vielmehr paradox werden [1]: Die Vielfalt erfordert organisationsweite Entscheidungen, behindert sie aber zugleich. Denn gerade bei Entscheidungen für das Gesamtspital, die über Klinikund Bereichsgrenzen hinausgehen, sind oft weder die Kriterien für Entscheidungen geklärt, noch die Art und Weise, wie entschieden wird oder wer in die Entscheidung eingebunden wird.

Im folgenden Beitrag loten wir diese Kernherausforderung von Spitalmanagement aus. Wir illustrieren die Paradoxie von Management anhand der unterschiedlichen Formen der Entscheidungspraxis. Von dieser «paradoxalen» Warte aus gesehen ist Management eigentlich eine unmögliche Aufgabe, die aber doch gelingt. Welche Möglichkeiten es dafür gibt, ist das Thema des zweiten und des dritten Beitrags in

\section{Sur l'impossibilité de gérer un hôpital - le paradoxe du management}

Gérer un hôpital est une tâche particulièrement complexe qui nécessite de tenir compte à la fois des multiples formes de prises de décisions des différentes professions et disciplines médicales tout en gardant à l'esprit l'hôpital dans son ensemble. C'est là tout le paradoxe du management, auquel $s$ 'intéresse la première partie de notre article. Paradoxe qui consiste à prendre des décisions pour toute l'organisation en dépit et à cause des multiples formes de prises de décisions de domaines partiels relativement autonomes.

dieser Reihe. Unter Management verstehen wir dabei nicht eine Stelle (z.B. den CEO) oder ein Gremium (z. B. die Geschäftsleitung). Management meint stattdessen die Funktion des Spitals, in einem überaus komplexen, vielfältigen und verteilten Wertschöpfungssystem für Entscheidungen zu sorgen, um eine kohärente und anspruchsgruppengerechte Leistung zu erbringen. Management zielt darauf ab, die kollektive Entscheidungs- und Handlungsfähigkeit des Spitals zu gewährleisten und weiterzuentwickeln.

\section{Die Vielfalt von Entscheidungsformen im Spital}

Die vielfältige Praxis des Entscheidens lässt sich treffend mit dem folgenden Witz illustrieren:

«Ein Anästhesist, ein Internist, ein Chirurg und ein Pathologe gehen zusammen auf Entenjagd. Es ist Entensaison, andere Vögel müssen jedoch geschont 
* Der Begriff «Administration» ist zunehmend unangemessen, da sich Abteilungen wie Finanzen, Betrieb, Infrastruk tur, Informationstechnologi oder Personal in den letzten Jahren grundlegend

verändern. Bislang hat sich noch keine neue Begrifflichkeit etabliert, die ihre Leistung in der fortlaufenden Bereitstellung zentraler Voraussetzungen für eine gelingende Behandlungsarbeit widerspiegelt. Deshalb werden diese Abteilungen hier mit «Systemlogistik» zusammengefasst. werden. Zuerst sieht der Anästhesist den Schwarm vorbeifliegen, er schaut kurz, meint: Jaaa, langer Hals, schwer zu intubieren. Das müssen Enten sein.> Schuss ... daneben. Beim nächsten Schwarm holt der Internist sein Fernglas raus, analysiert ganz genau: `Hmmm. Das sind genau sechs Tiere in V-Formation. Flugrichtung Nord-Nordost. Ein weibliches Tier an der Spitze, zwei männliche an den Positionen 2 und 3 sowie drei weitere weibliche Tiere, schätzungsweise 3 Jahre alt. Das sind höchstwahrscheinlich europäische Stockenten, aber ohne Differentialdiagnose. Ich werde nun also die zweite Ente von links erlegen. Gibt es Ihrerseits irgendwelche Einwände, meine Herren Kollegen? ... Nein? Also ... Doch bevor er schiessen kann, ist der Schwarm über alle Berge. Als nach 10 Minuten der nächste Schwarm erscheint, fackelt der Chirurg nicht lange. Er reisst die Schrotflinte hoch - BAMM BAMM - holt den ganzen Schwarm vom Himmel und sagt zum Pathologen: «Geh mal gucken, ob da 'ne Ente dabei ist!»»

Der Witz deutet die unterschiedlichen Bearbeitungsformen und damit die unterschiedliche Entscheidungspraxis verschiedener Disziplinen an. Diese Variationen haben sich im Zuge des medizinischpflegerischen Fortschritts historisch entwickelt und sind jeweils funktional, d.h. typisch für die unterschiedliche Behandlungspraxis (hier) ärztlicher Fachdisziplinen. Die schiere Anzahl dieser Fachdisziplinen ist vor allem seit dem zweiten Weltkrieg gestiegen. Heutzutage listet die FMH 45 Fachgesellschaften auf, die in eigener Weise ihre spezifischen medizinisch-pflegerischen Aufgaben im Rahmen der Patientenbehandlung wahrnehmen. Die Spezialisierung spiegelt den medizinisch-fachlichen Fortschritt und damit auch die Leistungsfähigkeit in der Behandlung von Patientinnen und Patienten wider. Fachgesellschaften, genauso wie Forschungseinrichtungen, Konferenzen, Journals usw. dienen der weiteren Entwicklung des Behandlungswissens, der Steuerung dieses Fortschritts sowie der Aus- und Weiter- bildung von HealthCare Professionals. Derartige Institutionen prägen die Professionszugehörigkeit einzelner Vertreterinnen und Vertreter.

Diese professionale und disziplinäre Prägung und die Vielfalt unterschiedlicher Fachrichtungen kommt im Spital zusammen. Glouberman und Mintzberg [2] unterscheiden vier immanente Welten, die der Heilung, der Pflege, der Handhabung knapper Ressourcen und die des lokalen Spitalumfeldes. Jede dieser Welten steht für ein eigenes Verständnis, priorisiert eigene Ziele und funktioniert in einer eigenen Weise zur Bearbeitung ihrer je eigenen Aufgabe. Nach unseren Beobachtungen ist vor allem die Welt der Heilung mit den ärztlichen Fachdisziplinen weitaus komplexer, als Aussenstehende oft meinen: Untersucht man die Entscheidungspraxis unterschiedlicher Fachdisziplinen in Hinblick darauf, wer über was wann und wie entscheidet, dann finden sich ganz unterschiedliche Formen des Entscheidens. Die folgende Tabelle enthält illustrativ und ausgewählt die Fachdisziplinen von Chirurgie und innerer Medizin, sowie die im Witz vernachlässigte Pflege und die sogenannte Administration, die wir «Systemlogistik» nennen*:

Chirurgische Entscheidungen sind auf Schnelligkeit ausgerichtet, werden damit tendenziell eher von Einzelpersonen getroffen und beinhalten im Anschluss an eine Intervention oder Handlung je nach Bedarf eine zügige Nachbearbeitung ggf. auftretender Nebeneffekte. Der Fokus dessen, was zu einem Entscheidungsproblem gehört, ist entsprechend eng auf eine gleichermassen zugeschnittene Aufgabe hin ausgerichtet, auf das akute vordergründige Problem. Dies ist sowohl am Operationstisch wie auch bei organisationalen Themen der Fall. Chirurgische Entscheidungen korrespondieren mit der originären Behandlungspraxis, die auf hochanspruchsvolle invasive Eingriffe fokussiert, die zeitkritisch sind und stark von der individuellen handwerklichen Fähigkeit des Operateurs abhängt.

\section{Tabelle 1}

Bereichsspezifisches Entscheiden. Vielfältige Formen der Entscheidungspraxis: 4 Beispiele aus einem Projekt.

\begin{tabular}{|c|c|c|c|c|}
\hline & Systemlogistik & Chirurgie & Pflege & Innere Medizin \\
\hline Wie & $\begin{array}{l}\text { Formalisiertes, berichts- } \\
\text { orientiertes Verfahren }\end{array}$ & $\begin{array}{l}\text { Aktion und } \\
\text { Nachbearbeitung }\end{array}$ & $\begin{array}{l}\text { Vordefinition der Aufgabe } \\
\text { und Einbezug der Basis } \\
\text { für die konkrete Umsetzung }\end{array}$ & $\begin{array}{l}\text { Fortlaufende Abklärung } \\
\text { (Abwarten) und Aktivität, } \\
\text { wenn die Zeit reif ist }\end{array}$ \\
\hline Wer & $\begin{array}{l}\text { Projektteam/Auftragnehmer; } \\
\text { fortlaufende Formalisierung } \\
\text { durch die GL }\end{array}$ & $\begin{array}{l}\text { Einzelperson (Chefarzt) } \\
\text { (ggbfls. nach Konsultation } \\
\text { mit Direktunterstellten) }\end{array}$ & $\begin{array}{l}\text { Pflegeleitung bis Stations- } \\
\text { mitarbeitende mit je unter- } \\
\text { schiedlichen Aufgaben }\end{array}$ & $\begin{array}{l}\text { Leitende (Gruppe) } \\
\text { möglichst im Konsens } \\
\text { etwaig betroffener Kollegen }\end{array}$ \\
\hline Was & $\begin{array}{l}\text { Fragestellung } \\
\text { einer Abteilung }\end{array}$ & Konkretes Problem & $\begin{array}{l}\text { Fragestellung und sozialer } \\
\text { Kontext (Atmosphäre, } \\
\text { Schnittstellen) }\end{array}$ & $\begin{array}{l}\text { Fragestellung und } \\
\text { Auswirkungen auf } \\
\text { betroffene Bereiche }\end{array}$ \\
\hline Wann & $\begin{array}{l}\text { Durch Auftrag, gemäss } \\
\text { geplantem Ablauf }\end{array}$ & $\begin{array}{l}\text { Ad hoc, } \\
\text { schnelle (Re-)aktion bei } \\
\text { Gelegenheiten }\end{array}$ & $\begin{array}{l}\text { Durch Auftrag, gemäss } \\
\text { geplantem und sich } \\
\text { abzeichnendem Verlauf } \\
\text { bei Umsetzung }\end{array}$ & $\begin{array}{l}\text { Bei sich abzeichnenden } \\
\text { Themen; } \\
\text { Antwort auf Gelegenheiten }\end{array}$ \\
\hline
\end{tabular}


Innere Mediziner entscheiden demgegenüber vergleichsweise zeitintensiver mit explizitem Einbezug von Kolleginnen und Kollegen, mit denen idealerweise ein Konsens erzielt wird. Diese Vorgehensweise orientiert sich an einem wesentlichen Kern ihrer medizinischen Tätigkeit, dem Stellen von Diagnosen. Vor dem Hintergrund oft vielfältiger und zeitlich veränderlicher Symptome gilt es Muster zu erkennen. Diese Muster werden in meist konsenualen Absprachen unter den Kolleginnen und Kollegen auf bestimmte Hypothesen hin verdichtet. In analoger Weise lässt sich die Bearbeitung organisationaler Fragestellungen beobachten. Entsprechend dieser Art des Entscheidens ist der Fokus, was zu einer Entscheidungssituation gehört, vergleichsweise breiter gefasst und umfasst sowohl die zeitliche Dimension als auch die Perspektive potentiell Betroffener bei organisationalen Fragestellungen.

Entscheidungen in der Pflege beinhalten die physiologische, die psychologische und die soziale oder relationale Dimension zwischen den Beteiligten. Dies spiegelt einen Kern der Pflegetätigkeit wider, für

\section{Die Entscheidungspraxis der verschiedenen}

\section{Fachdisziplinen unterscheidet sich erheblich im Hinblick darauf, wer über was wann und wie entscheidet.}

Interaktiver
Artike:
Wollen Sie diesen Artikel
kommentieren? Nutzen
Sie dafür die Kommentar-
funktion in der Online-
Version oder sehen Sie
nach, was Ihre Kolleginnen
und Kollegen bereits
geschrieben haben:
www.saez.ch/
aktuelle-ausgabe/
interaktive-beitraege/

kranke Menschen während ihres Aufenthaltes bis hin zur Vorbereitung des Austritts Sorge zu tragen. Analog werden Entscheidungen und Entwicklungsbestrebungen für organisatorische Fragen möglichst breit über unterschiedlichen Hierarchiestufen durch Einbindung abgestützt. Ab dem mittleren Kader nehmen Pflegefachkräfte verstärkt oder ausschliesslich Führungs- oder Managementaufgaben wahr. Entsprechend kommen auch verstärkt Management-

Instrumente zum Einsatz. Zudem ist ein fortlaufendes Einbinden von Stationsteams zu beobachten, mit denen die konkrete Umsetzbarkeit entwickelt und eine positive Atmosphäre auf der Station angestrebt wird.

Entscheidungen der Systemlogistik sind im Vergleich zu den fachlichen Professionen schwerer zu beschreiben. Dieser Bereich durchläuft derzeit eine fundamentale Weiterentwicklung von einer traditionell eher bürokratischen zu einer eher unternehmerisch-orientierten Entscheidungspraxis. Gleichzeitig steht die Systemlogistik insbesondere gegenüber der Ärzteschaft und Pflege in einem besonderen Rechtfertigungsdruck, weil sie selbst nicht auf eine Behandlungspraxis als Legitimation verweisen kann. Traditionell praktizierte die Spitaladministration Entscheidungen eher nach einem bürokratischen Modell, bei der die Einhaltung eines formalisierten Verfahrens im Vordergrund steht. So beschreibt ein
Mitarbeiter die Bearbeitung von interdisziplinären Projekten als Abfolge von Vor-, Zwischen- und Endberichten zuhanden der Geschäftsleitung. Entscheidungen werden entsprechend sequentiell vorab geplant, möglichst durch die Geschäftsleitung beschlossen, umgesetzt und anschliessend kontrolliert. Man kann dies, wie in unserem Fall seitens der Ärzteschaft als «Projektitis» bezeichnen, oder auch als Versuch verstehen, irgendwie mit den unterschiedlichen Interessen, Sichtweisen und Formen der Entscheidungspraxis umzugehen.

Diese idealtypische Darstellung deutet an, wie vielfältig die Entscheidungspraxis in Spitälern wirklich ist. Diese Vielfältigkeit entsteht und besteht fort, weil die tradierten Formen der Entscheidungspraxis aus der jeweiligen Behandlungsarbeit mit Patientinnen und Patienten auf organisationale Fragestellungen übertragen werden [3]. Die Übertragung auf organisationale Themen vermittelt ein hohes Mass an Legitimität und Selbstverständlichkeit für die jeweilige Entscheidungspraxis. So erläutert ein Chirurg die organisationale Entscheidungspraxis und hierarchische Ausrichtung auf den Chefarzt in seiner Klinik: «Am Ende steht einer am Tisch und führt den Schnitt.»

\section{Die Paradoxie von Management im Spital}

Die Vielfältigkeit der Formen von Entscheidungspraxis bildet einen massgeblichen Hintergrund für die gegenwärtige Bearbeitung organisationsweiter Fragestellungen.

Spätestens mit der Einführung der DRGs, dem Wegfall der Kantonsgrenzen sowie der Defizitübernahme bei öffentlichen Spitälern treten heutzutage vermehrt Entscheidungsnotwendigkeiten auf, die über die historisch gewachsenen Grenzen relativ autonomer Fachbereiche, Kliniken und Abteilungen hinausgehen und das Gesamtspital betreffen [3]: Mit Blick nach aussen geht es zum Beispiel um die Positionierung des Spitals insgesamt mit einem spezifischen Angebot an Behandlungsfeldern und Dienstleistungen, was mitunter den Abbau oder das verlangsamte Wachstum anderer Fachgebiete beinhaltet. Verstärkt sind damit auch Kooperationen mit anderen Leistungsanbietern gefragt, eine besondere Gestaltung der Zuweiserbeziehungen bis hin zur Entwicklung integrierter Versorgungsregionen.

Gleichzeitig und innerhalb des Spitals wird vermehrt die Herausforderung wahrgenommen, geeignetes Fachpersonal zu rekrutieren und zu binden. Darüber hinaus verlangt die fachlich notwendige Spezialisierung eine verstärkte Re-Integration von Behandlungspfaden. Das bedeutet einen erhöhten Abstimmungs- und Koordinationsbedarf zwischen Professionen und Disziplinen, gehandhabt beispielsweise über die Einrichtung von Zentren oder bei der Durchführung von Initiativen zur Prozessoptimierung. Wie der Leiter eines grossen Kantonsspitals erläutert, kümmerten sich in den 70er Jahren schätzungsweise etwa 2,5 Fachpersonen um einen Patient. 
Abbildung 1

Paradoxie von Management.

\section{Paradoxie «multirationales Management»}

Ebene der Kliniken/Fachbereiche/Abteilungen vs. Ebene Gesamtspital

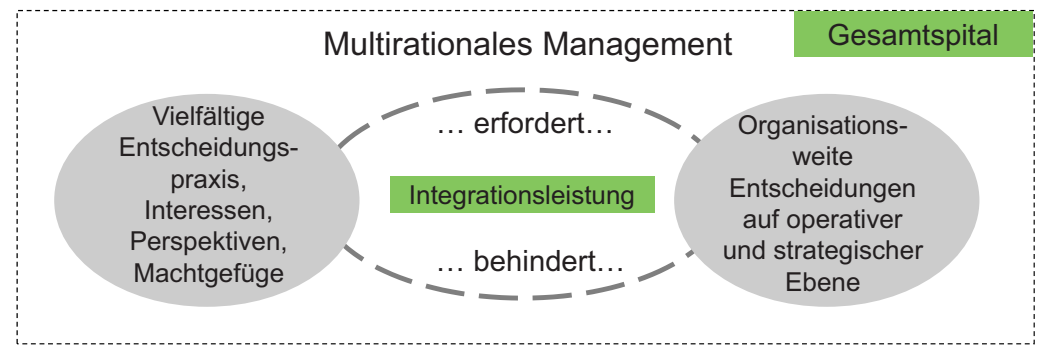

In den 90er Jahren seien es 15 gewesen, Tendenz weiter steigend. Bereichsübergreifende bzw. organisationsweite Entscheidungen gewinnen daher aufgrund des medizinisch-pflegerischen Fortschritts enorm an Bedeutung. Denn letztlich sind die Leiden von Patientinnen und Patienten nur bedingt nach Fachrichtungen aufteilbar.

Diese Zunahme und Bedeutung organisationsweiter Entscheidungen und die vielfältige Entscheidungspraxis in Spitälern bilden die zwei Seiten oder

\section{Spitäler haben ganz unterschiedliche Stossrichtungen entwickelt, um mit der Paradoxie umzugehen.}

Pole der Paradoxie von Management im Spital. Die Paradoxie von Management im Spital besteht darin, im Kontext des Gesamtspitals (symbolisiert durch das gestrichelte Rechteck in der folgenden Abbildung) für organisationsweite Entscheidungen zu sorgen. Diese organisationsweiten Entscheidungen werden einerseits erforderlich aufgrund der unterschiedlichen Bereiche, die jeweils eigene Perspektiven einnehmen, eigene Interessen verfolgen $[4,5]$ und jeweils eine eigene Form des Entscheidens praktizieren. Andererseits und zugleich behindert diese Vielfalt organisationsübergreifende Entscheidungen. Denn die jeweiligen Bereiche verfolgen unterschiedliche Interessen, nehmen eine jeweils andere Perspektive ein und entscheiden in einer anderen Art und Weise. Management als Funktion der Organisation ist daher eine Integrationsleistung, die wegen der Vielfältigkeit nötig und gleichzeitig schwierig ist. Das ist die Paradoxie von Management im Spital.

Im Zuge der jüngsten Entwicklungen im Gesundheitssektor wird diese Paradoxie weiter an Bedeutung gewinnen. Denn auf der einen Seite ist mit dem medizinisch-fachlichen Fortschritt, der demographischen Entwicklung und anderer Faktoren davon auszugehen, dass die Möglichkeiten und Optionen medizinischer Behandlung weiterhin zunehmen und damit auch der Fortschritt durch Spezialisierung. Gleichzeitig ist aber zu beobachten, dass die dafür notwendigen monetären, personellen, infrastrukturellen und technischen Ressourcen in einem Spital nicht in gleicher Weise steigen. Daraus entsteht eine Schere zwischen dem, was möglich ist und dem, was auch realisierbar ist. Mit dieser Schere steigt der Bedarf an bereichsübergreifenden Fragestellungen.

\section{Management von Spitälern: Handhabung einer Paradoxie}

Vor dem Hintergrund dieser Paradoxie wird Management zu einer hochanspruchsvollen Aufgabe, die zwar nicht unmöglich, aber paradox ist. Eine einzelne Person, eine Stelle, oder eine Abteilung kann diese Aufgabe kaum bewältigen. Vielmehr erfordert Management, das Repertoire an vielfältigen Formen des Entscheidens ernst zu nehmen und gleichzeitig die übergreifenden, organisationsweiten Fragestellung des Gesamtspitals im Blick zu behalten. Management ist daher etwas, was auf allen Ebenen des Spitals stattfindet, von täglichen Abstimmungen zu Behandlungsfragen über die Zusammenarbeit zwischen Professionen und Disziplinen auf Stationen, über grösser angelegte Verknüpfungen beispielsweise bei der Einrichtung von Behandlungszentren bis hin zur Gesamtausrichtung des Spitals mit der Definition seiner Leistungsangebote und Dienstleistung sowie seiner Einbettung in das eigene Umfeld.

Spitalmanagement als Paradoxie verstanden verdeutlicht, dass sich die darüber dargestellte Herausforderung nicht wegdiskutieren oder auflösen lässt. Das bedeutet aber auch nicht, vor der Paradoxie die Augen $\mathrm{zu}$ verschliessen oder zu kapitulieren. Vielmehr haben Spitäler ganz unterschiedliche Stossrichtungen entwickelt, um mit der Paradoxie umzugehen. Das ist das Thema der folgenden beiden Beiträge.

\section{Literatur}

1 Ortmann, G. Schmuddelkinder der Logik. Berliner Debatte Initial. 2004;15(1):18-27.

2 Glouberman S, Mintzberg H. Managing the care of health and cure of disease - part I: Differentiation. Health Care Management Review. 2001;26(1):56-70.

3 Tuckermann H, Rüegg-Stürm J. Führung im Spital: Weiterentwicklung der eigenen Entscheidungspraxis auf dem Weg in die Zukunft. Swiss Review of Military and Disaster Medicine. 2012;2:32-9.

4 Denis JL, Lamothe L, Langley A. The Dynamics of Collective Leadership and Strategic Change in Pluralistic Organizations. Academy of Management Journal. 2001;44(4):800-37.

5 Denis JL, Langley A, Rouleau L. Strategizing in pluralistic contexts: Rethinking theoretical frames. Human Relations. 2007;60(1):179-215. 